
centrasiatiques et tibétaines

$32 \mid 2001$

L'épopée orale turque d'Asie centrale. Contes épiques nanaî̀s

\title{
III. Le héros et le chamane
}

Les strates archaïques de l'épopée turque

III. Hero and Shaman: Archaic Layers of the Turkic Epic

\section{Karl Reichl}

\section{(2) OpenEdition}

\section{Journals}

Édition électronique

URL : https://journals.openedition.org/emscat/1287

DOI : 10.4000/emscat.1287

ISSN : 2101-0013

Éditeur

Centre d'Etudes Mongoles \& Sibériennes / École Pratique des Hautes Études

Édition imprimée

Date de publication : 1 décembre 2001

Pagination : 69-95

ISBN : 2-9518888-0-5

ISSN : 0766-5075

Référence électronique

Karl Reichl, « III. Le héros et le chamane», Études mongoles et sibériennes, centrasiatiques et tibétaines

[En ligne], 32 | 2001, mis en ligne le 17 mars 2009, consulté le 13 juillet 2021. URL : http:// journals.openedition.org/emscat/1287 ; DOI : https://doi.org/10.4000/emscat.1287

Ce document a été généré automatiquement le 13 juillet 2021.

(c) Tous droits réservés 


\title{
III. Le héros et le chamane
}

\author{
Les strates archaïques de l'épopée turque \\ III. Hero and Shaman: Archaic Layers of the Turkic Epic
}

\section{Karl Reichl}

Dans un article consacré à la magie et à l'exorcisme chez les Kazakhs et autres peuples turcs d'Asie centrale, paru dans la Revue des études islamiques en 1930, J. Castagné cite le poème suivant, auquel il donne le titre "Évocation des saints ${ }^{1}$ :

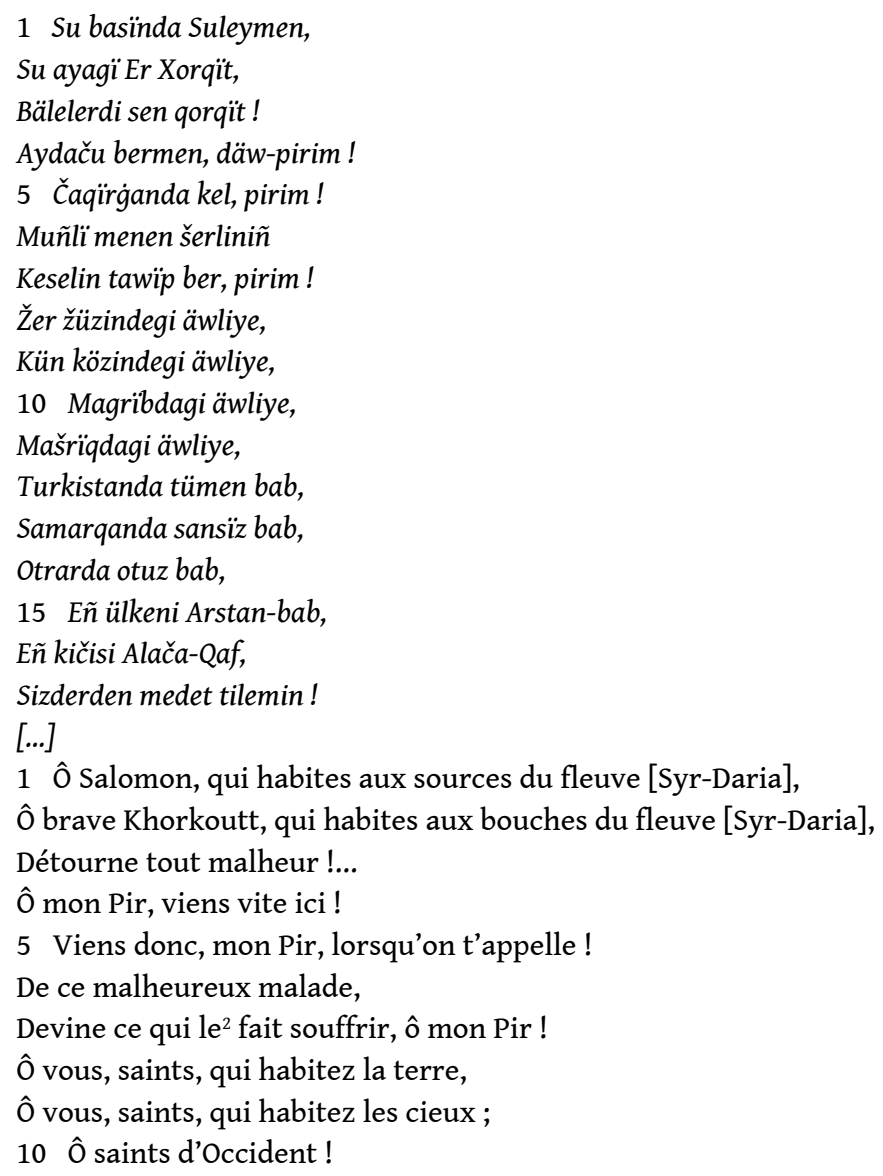


Et vous saints d'Orient!

Ô vous, dix mille saints du Turkestan,

J'implore votre secours!

Ô innombrables saints de Samarkande,

15 Et vous, trente saints d'Otrar ;

Le plus ancien d'entre eux est Aryslan-bâb,

Et le plus jeune - Alatcha-kaf :

J'implore votre secours!

[...]

2 Le poème (qui compte quarante-six vers) se poursuit avec l'invocation de plusieurs saints, dont les šilten, les quarante sauveurs-patrons des peuples turcs islamisés ${ }^{3}$. Ce poème pourrait être extrait d'une épopée comme Qoblan où le héros implore l'aide de son pir et des saints islamiques. Même la métrique est celle de l'épopée : les vers comportent sept ou huit syllabes; ils sont rimés ou assonancés, la rime (ainsi que l'allitération) résultant surtout du parallélisme stylistique ${ }^{4}$. L'invocation des esprits est exprimée en des termes conformes à l'islam populaire. Un premier coup d'œil ne révèle aucun trait chamanique altaïque ou sibérien.

Un des saints auquel il est fait appel dans ce poème est le «brave Khorkoutt » (Er Xorqït). Nous avons déjà rencontré brièvement Qorqut-Ata en relation avec Baba Qambar, qui chez les Turkmènes est vénéré comme patron de la musique et du chant. Dans le Kitab-i Dedem Qorqut, "Le Livre de mon grand-père Qorqut ", Qorqut est un barde qui raconte au khan les aventures des Oghouzes ${ }^{5}$ :

Dedem Qorqut geldi, šadiliq čaldï, boy boyladï, soy soyladï, ġāzi erenler bašïna ne geldügin söyledi, bu Og்uz-nāme Beyregüñ olsun didi.

Mon grand-père Qorqut venait, jouait gaiement [du qopuz], récitait l'histoire, chantait les vers, racontait les aventures des braves guerriers et nommait ce récit des Oghouzes celui de Beyrek.

De semblables formules se rencontrent à la fin de la plupart des récits qui constituent le «Livre de mon grand-père Qorqut ». Chez les Kazakhs, Qorqut-Ata est aussi un barde, mais il est en outre - comme Baba Qambar chez les Turkmènes - le patron de la musique et des bardes. Selon la tradition kazakhe, Qorqut est le premier barde des Turcs.

5 Un grand nombre de légendes ont été collectées, dont des " légendes musicales ». Chez les Kazakhs, une tradition veut que différentes mélodies soient associées à une même légende. Ces mélodies (généralement pour la dombïra), nommées küy, ont souvent un caractère imitatif de mouvements ou de sons naturels ${ }^{6}$. Une des légendes musicales ayant trait à Qorqut-Ata explique son rôle d'éternel musicien. Un jour, Qorqut-Ata ressentit l'approche de la mort et décida de s'enfuir. Monté sur son chameau Želmaya, Dromadaire-Vent, il s'enfuit jusqu'au bout du monde. Là, il vit des hommes qui creusaient une tombe. Qorqut-Ata les interroge sur l'identité du mort ; ils lui répondent que le tombeau est pour un homme qui s'appelle Qorqut. Épouvanté, Qorqut-Ata reprend sa fuite, mais la même histoire se répète plusieurs fois. Comprenant enfin qu'il faut mourir, Qorqut-Ata se résigne à l'inévitable. Selon une version de la légende, il joue de la dombïra et exprime son chagrin dans son chant. C'est ainsi qu'il lègue ses mélodies et chansons à la postérité : "Même si le brave homme meurt, ses mots ne meurent pas,/ L'immortelle voix de la vie reste après lui !" (Žaqsiliñ özi ölse de sözi ölmeydi,/ Artïnda öšpes ömir üni qaldii) ${ }^{7}$. Selon une autre version, Qorqut-Ata échappe d'abord à la mort en flottant sur son tapis magique au-dessus du Syr-Daria et en jouant du qobïz. 
Quand il finit par mourir, le qobïz est mis dans sa tombe, d'où sort encore aujourd'hui le son de sa musique ${ }^{8}$.

Dans notre texte, Qorqut-Ata est invoqué dès le début du poème, ce qui souligne son importance. Un autre poème, publié lui aussi par Castagné, est encore plus explicite ${ }^{9}$ :

Su ayag̈i Er Xorqït,

Bäleketti sen qorqüt!

Baqsï piri emespeñ?

Köziñdi sal, qolum tut!

Baqsï baba sen qolda!

Ô brave Khorkoutt, qui habites le bassin inférieur du Syr-Daria,

Fais peur à la maladie [du malade] :

N'es-tu point le pir du baqça?

Porte tes yeux vers moi, aide-moi,

Ô baqça baba, prête ton appui!

7 Comme on le voit, Qorqut-Ata n'est pas seulement le pir des bardes, il est aussi celui des baqsï. Nous avons déjà rencontré ce mot - sous les formes baxši, baxši, baqsï - en tant qu'appellation du barde en ouzbek, en ouïghour, en turkmène et en karakalpak. Mais chez les Kazakhs - comme d'ailleurs chez les Kirghizes - baqsï (baqši) signifie le chamane, ou bien son descendant moderne, le guérisseur et praticien de médecine populaire ${ }^{10}$. Cette différence de sens dans des langues apparentées peut surprendre. Mais c'est en réalité un des nombreux indices qui laissent supposer que, dans les traditions turques, barde et chamane ont une origine commune. En effet, en ouzbek et en ouïghour, baxši désigne le barde aussi bien que le chamane. (Rappelons qu'en kirghize et en kazakh il y a d'autres termes pour désigner le barde, tels žiršï, aqün, manasči, džomoqču, etc.) En turkmène et en karakalpak, le chamane est appellé porxan, terme dérivé (par l'intermédiaire du chinois) du sanskrit buddha, "l'éveillé », et qui signifie «Dieu », « idole » dans d'autres langues turques ${ }^{11}$. Dans les langues turques de l'Altaï ainsi qu'en turc ancien, le chamane est appellé qam, xam, terme d'origine inconnue dont dérive le russe kamlenie, "séance chamanique $»^{12}$.

8 Le chamane est la figure centrale du chamanisme, cet ensemble de croyances et de pratiques qui est la marque de la vie spirituelle des peuples indigènes de Sibérie. Il est hors de ma compétence d'approfondir ici la question du chamanisme en tant que phénomène religieux. Seules les traces laissées par le chamanisme dans l'épopée orale des peuples turcs nous occuperont ici. Pour les retrouver, il me semble utile de rappeler brièvement la conception du monde chamanique et le rôle du chamane :

Le chamanisme implique une vision intangible de l'univers conçu comme divisé en deux ou trois zones superposées, le ciel et la terre ou le ciel, la terre et le sous-terre, que relie un axe cosmique permettant le passage éventuel de l'une à l'autre; et l'existence d'une multitude de personnages invisibles mais zoomorphes, omniprésents dans les deux ou trois étages cosmiques et dans les régions qui les séparent, auxquels on peut donner par commodité le nom d'esprits. Il repose sur une technique archaïque de l'extase que vise à atteindre, par la transe, son seul spécialiste, le chaman. [...]

La séance chamanique n'est pas effectuée par désintéressement ou égoïsme (pour obtenir des états mystiques personnels), mais, comme je l'ai dit, pour des raisons bien déterminées. Il s'agit fondamentalement d'interroger les esprits sur les secrets qu'ils possèdent, c'est-à-dire de prédire l'avenir, de rechercher l'âme des malades, âme dérobée par des êtres invisibles ou vagabonde et menacée par eux d'enlèvement, c'est-à-dire de procéder à la guérison magique. ${ }^{13}$ 
Dans les traditions turques d'Asie centrale, le barde et le chamane sont deux figures bien distinctes, même s'ils peuvent être désignés par un même terme. En revanche, l'union du chamane et du barde en une même personne n'est pas inconnue du monde sibérien. L. Ja. Šternberg publia au début du $\mathrm{xx}^{\mathrm{e}}$ siècle une riche collection de textes folkloriques recueillis chez les Ghiliaks (Nivkhs), peuple peu nombreux (3 690 individus en 1959) qui parle une langue paléoasiatique et habite le bassin inférieur de l'Amour et le nord de l'île de Sakhaline. Dans la préface de son recueil, il donne des renseignements précieux sur les bardes :

Le barde ghiliak a une nature exceptionnelle, c'est un véritable élu des dieux. Il n'est pas fortuit que les bardes soient dans la plupart des cas des chamanes ou des descendants de chamanes. On dit généralement de chamanes ghiliaks célèbres qu'en plus de leurs diverses actions surnaturelles, ils passaient des jours entiers à conter des récits et à chanter des poèmes improvisés. Il y a naturellement des exceptions, mais de par leur nature psychique barde et chamane sont apparentés. Tous deux - de tempérament maladif, nerveux, hystérique et enclin à tomber facilement en extase - possèdent le don de vision; tous deux croient en leur élection et possèdent leurs esprits-protecteurs personnels. ${ }^{14}$

10 L'indice le plus clair de cette parenté est leur vocation « mystique ». Quant à l'élection et à l'initiation du chamane, je renvoie à la littérature sur le chamanisme en général, et surtout aux textes réunis par G. V. Ksenofontov sur les chamanes iakoute, bouriate et toungouse ${ }^{15}$. Plus intéressantes pour notre propos sont les légendes d'élection des bardes. Il en existe chez les Turcs de Turquie comme chez ceux d'Asie centrale et de Sibérie $^{16}$. Ainsi, le célèbre manasči Sağïmbay Orozbaqov raconte cette vision : un jour, toute sa famille tomba malade de la variole. En proie à la fièvre, le futur barde voit une yourte énorme dans la plaine de Qočqor. Il entre dans la yourte. Là se trouvent des hommes d'une stature gigantesque qui le prient de s'asseoir à la place d'honneur et de chanter l'épopée. Sagï̈mbay refuse, et un homme, somptueusement vêtu, sans doute Semetey, le fils de Manas, le menace avec une hache de guerre. Sag̈ïmbay s'aperçoit alors que dans la yourte se trouvent plusieurs bardes qui narrent différentes parties de l'épopée: l'un raconte la naissance de Manas, un autre l'histoire de Közkaman et un autre encore la grande expédition à Pékin. Peu après Sagïmbay se réveille. Sa famille et lui guérissent de la variole, et il devient par la suite un grand barde ${ }^{17}$. Dans ces visions, l'un des héros importants du cycle épique apparaît au barde et lui commande de chanter son épopée. La vision peut avoir lieu en songe, ou bien au cours d'une maladie. Cela évoque les visions du chamane lors de son initiation et de la maladie qui souvent accompagne cette initiation.

11 Un autre phénomène encore pourrait être considéré comme rapprochant barde et chamane. Les ethnomusicologues ont noté chez beaucoup de peuples non occidentaux une manière artificielle de chanter, souvent liée au chamanisme. «Partout où le chant est un acte d'extase et de dépersonnalisation, il s'éloigne de l'expression humaine naturelle ", écrit Curt Sachs dans son livre sur les origines de la musique ${ }^{18}$. Et Gilbert Rouget, dans son étude sur la musique et la transe, souligne à plusieurs reprises le «travestissement» de la voix du chamane lors des séances chamaniques ${ }^{19}$. Il est intéressant de remarquer que ce même "travestissement" s'applique aussi à la manière de chanter l'épopée dans certaines traditions turques. Je pense surtout au style qu'utilise le žiraw karakalpak. Il chante l'épopée d'une voix "serrée", étranglée, glottale, très éloignée de la qualité de voix qui caractérise sa récitation de la prose. Un « travestissement » semblable, mais d'une qualité différente, est employé par les bardes 
altaïens. Ce chant, appelé qay, est une sorte de chant pharyngal ("chant de gosier »), dans lequel les notes ne sont pas articulées d'une manière ouverte, mais plutôt d'une manière fermée et resserrée ${ }^{20}$.

Il me semble bien possible que cette façon de chanter marque l'altérité du barde, son état d'exaltation et d'inspiration poétique, et qu'elle prenne ses racines dans le chant d'extase du chamane. Le chant qay des Altaïens est tout à fait analogue à la manière de chanter des Bouriates, laquelle serait, selon Roberte Hamayon, à rapprocher du chamanisme ${ }^{21}$ :

Un détail vocal souligne ce caractère en quelque sorte transitoire de l'exécution épique, qui fait d'elle un pont entre les modes d'adresse aux esprits animaux respectifs du chamane - qui imite leur nature animale - et du chasseur - qui leur parle comme à des humains. Le mode de chanter consiste à ancrer la voix dans la production d'un son de bourdon d'où naissent, sans que soit repris le souffle, des sons articulés, les syllabes des premiers vers de l'épopée, puis de chacune de ses subdivisions. Puisque le bourdon se veut imitation de sons animaux, ce mode de chanter réalise en somme le passage de l'animal à l'humain au sein de la même émission de voix.

La musicologue polonaise Slawomira Zerańska-Kominek a aussi attiré l'attention sur la manière de chanter du baxši turkmène. D'après ses observations, le barde passe par trois stades émotionnels successifs comparables au déroulement d'une séance chamanique. Ceci se marque, musicalement, par une montée d'un registre bas à un registre haut ; une même montée se laisse aussi entendre chez certains bardes ouzbeks. Ils entonnent les passages chantés d'une voix étranglée, dans des registres bas, et la voix s'élève au fur et à mesure du chant vers des registres plus hauts pour aboutir à une déclamation non mélodique. Le barde est dit alors «bouillir » (qaynamāq) 22 . Cependant, il faut apporter un certain nombre de restrictions à cette thèse. En effet, chanter l'épopée selon ce style n'est pas caractéristique de toutes les traditions turques : quand on étudie les manières d'exécuter l'épopée orale des différents peuples turcs, on est frappé par la grande variété qu'on y trouve. Il faut donc se garder de toute généralisation. Par ailleurs, il existe des enregistrements de chants chamaniques sibériens qui ne sont nullement marqués par le style qu'on associe en général au chant du chamane ${ }^{23}$.

La musique épique peut être reliée au chamanisme aussi par l'instrument dont s'accompagne le barde. Le qobïz qu'utilise dans le rite le baqsï (guérisseur) kazakh joue le même rôle que le tambour dans d'autres traditions chamaniques, c'est-à-dire qu'il est employé comme moyen (symbolique) de transport depuis le monde moyen vers les mondes supérieur et inférieur. Comme le remarque Castagné, « le kobouz, de même que le tambourin, est considéré comme un instrument doué d'une puissance magique ; on le dit même susceptible de s'animer $»^{24}$. Or, ce qobïz est aussi l'instrument du barde (žiraw) karakalpak et, en certaines régions, du barde kazakh.

Outre les rêves d'initiation, la manière de chanter l'épopée et la terminologie employée pour le barde et le chamane, le chamanisme a également laissé des traces dans l'épopée elle-même. Il fournit l'arrière-plan mythologique des épopées des peuples turcs non islamisés. C'est ainsi que l'on peut déceler des traits d'une conception du monde chamanique dans l'épopée turque de l'Altaï et de la Sibérie (chez les Iakoutes) : dans sa structure narrative et son contenu, comme dans le choix et la peinture de ses protagonistes. Bien que voilée, une couche chamanique se laisse aussi discerner dans l'épopée des peuples turcs d'Asie centrale. Avant d'aborder l'influence du chamanisme 
sur l'épopée des traditions centrales, je caractériserai brièvement l'épopée altaïenne, c'est-à-dire des peuples turcs de l'Altaï. Ces peuples parlent tous des dialectes apparentés et utilisent comme langue littéraire l'altaïen proprement dit (appelé autrefois « ö̈rote ») $)^{25}$. Le nom de l'épopée y est čörčök, terme qui, comme le touvinien tool, désigne aussi bien le conte que l'épopée; le narrateur de conte est dit čörčökči, " conteur ", le barde qayči, car sa manière de chanter est celle du qay mentionné plus haut. En outre, le barde s'accompagne du topšuur, instrument à deux cordes, d'ordinaire pincées mais pouvant aussi être jouées à l'archet.

Les premiers čörčök furent publiés par Wilhelm Radloff en 1866 ; ce sont dix poèmes, dont les plus longs comportent presque mille vers ${ }^{26}$. En tout, on a recueilli environ 220 čörčök (y compris les variantes) et publié quelque 120 textes $^{27}$. S. S. Surazakov (1985) en propose une classification chronologique, qui distingue trois périodes : celle de la société tribale, celle de la formation des premières structures féodales et celle de la société patriarco-féodale, illustrées respectivement par : Altay Buučay, Maaday Qara et Kögütey. Cette classification en trois périodes pose problème et mérite discussion. Les différences entre les trois épopées citées, réelles, ne se laissent pas expliquer par ce schéma chronologique. Le fait que Kögütey, par exemple, soit un homme pauvre ne me semble pas suffisant, contrairement à ce que pense Surazakov, pour classer ce čörčök dans la période patriarco-féodale. Toutefois, même si l'on ne partage pas l'avis de Surazakov sur cette périodisation des čörčök altaïens, il faut concéder que son étude donne une analyse précieuse de l'œuvre épique de ce petit peuple. Pour en donner un aperçu, j'ai choisi Kögütey et Altay Buučay. Je présenterai d'abord un bref résumé de Kögütey d'après la version de M. Jutkanakov; c'est un čörčök d'environ 3000 vers qui fut mis par écrit en 1914 et publié (en altaïen avec traduction russe) pour la première fois dans les années 1933 à $1935^{28}$.

Kögütey est le nom d'un vieux qui, un jour, alors qu'il abattait un mélèze, sauve la vie à un castor qu'il adopte comme fils. Le castor prie son père adoptif d'aller demander la main de Temene-Qoo (Belle-Aiguille), fille cadette de Qaratï-qaan. Le castor va chercher de l'eau-de-vie pour donner courage à Kögütey. Kögütey est battu à mort par les six gendres du khan, mais le castor le ranime. Le même sort attend Kögütey lors de sa deuxième visite au palais du khan, mais le castor le ranime derechef. Pour sa troisième visite, le castor enivre Kögütey à un tel degré que le vieux se comporte en héros et demande hardiment au khan la princesse pour son fils. Le khan exige un prix de la fiancée exorbitant. Le castor arrive néanmoins à satisfaire le khan, et il épouse la princesse.

Suivent alors plusieurs tâches que le khan impose à ses gendres. Il leur commande d'abord d'aller à la recherche d'une jument pie, puis de ses six poulains. Le castor laisse sa peau à sa femme, lui défendant de la montrer à quiconque, et commence par aller attraper un cheval. Le cheval, un poulain noir comme jais, donne (au moyen d'une lettre attachée à son encolure) un nom au héros : Qusqun-Qara-Baatïr, Héros-noir-comme-un-corbeau. La lettre révèle aussi que le père du héros est la Forêt noire aux cent cascades et sa mère la Mer bleue aux cent baies. Qusqun-QaraBaatï se met en route et se dirige vers le nid de l'oiseau Qaan-Kerede (Garuda), qui avait enlevé les six poulains. Arrivé au nid, le héros aperçoit un serpent qui attaque les petits de l'oiseau. Qusqun-Qara-Baatïr tue le serpent et gagne ainsi la gratitude de Qaan-Kerede. L'oiseau remet les poulains au héros, et les nouveaux amis échangent des signes de vie (une flèche pour l'oiseau, une plume pour le héros).

À son retour, Qusqun-Qara-Baatïr est tué par ses beaux-frères, mais Qaan-Kerede, voyant la flèche se rouiller, accourt à son aide et le ranime. Qusqun-Qara-Baatïr punit ses beaux-frères et son beau-père, réprimande sa femme Temene-Qoo qui avait montré sa peau de castor à ses sœurs, brûle la peau et s'en retourne chez Kögütey et son épouse, qu'il rend jeunes et riches. 
Le pays de Qaratï-qaan se change en désert couvert de neige. Les hommes et les animaux meurent, et Temene-Qoo se met en route pour retrouver son mari. Elle arrive enfin à sa demeure, où elle est reçue amicalement par les deux femmes de Qusqun-Qara-Baatïr. Un peu plus tard le héros retourne voir l'oiseau Qaan-Kerede et sa fille cadette, Altïn-Jüstük (Anneau-d'or). Le récit finit par le mariage de Qusqun-Qara-Baatïr avec Altïn-Jüstük. Depuis ce temps-là, un été éternel règne sur l'Altaï.

Ce čörčök allie deux types d'éléments : d'un côté, des motifs que l'on trouve aussi dans les contes de fées et, de l'autre, des motifs qui appartiennent plus proprement au mythe. Du point de vue stylistique, ces motifs sont traités d'une manière qui relève plus du style de l'épopée que de celui du conte ${ }^{29}$. Sans en donner une analyse détaillée, je signalerai les éléments suivants. Une partie du récit est une version du conte d'Amour et Psyché (Aarne-Thompson, $\mathrm{n}^{\circ} 425 \mathrm{sq}$.), dont les unités les plus marquantes sont d'après l'analyse de J.-Ö. Swahn : le mari surnaturel ; le mariage et l'interdit de détruire la peau animale du mari (dans l'épopée, de montrer la peau); la violation de l'interdit (dans l'épopée la femme montre la peau à ses sœurs, et l'une d'elles y perce un trou); la disparition du mari et la quête de l'épouse ${ }^{30}$. Quant à la première partie du čörčök, l'adoption du castor, on y relève entre autres les motifs suivants: N825.1 (Un couple sans enfant adopte le héros), B313 (L'animal reconnaissant est une personne enchantée), B620 (Le prétendant est un animal) et B640 (Le mariage avec une personne à forme animale) ${ }^{31}$.

18 Une autre partie du récit comprend les tâches imposées au héros par son beau-père et la trahison de ses beaux-frères. Cet ensemble de motifs trouve son équivalent le plus proche dans le conte de la Quête de l'oiseau d'or (Aarne-Thompson, $n^{\circ} 550$ ). Pour ce conte, Aarne-Thompson énumère les motifs caractéristiques suivants : le vol d'un objet par un oiseau merveilleux (dans l'épopée, ce sont les six poulains dérobés par QaanKerede); la quête entreprise par les fils du roi (dans l'épopée, par les beaux-fils); la trahison des frères au retour du héros et la délivrance du héros par un animal reconnaissant. À cet ensemble s'entrelace une autre série de motifs, celle que l'on retrouve en particulier dans le conte de Jean de l'Ours (Aarne-Thompson, $\mathrm{n}^{\circ} 301$ ) : le héros, sous terre, délivre des princesses; au cours de ses aventures souterraines, il sauve aussi les petits d'un oiseau en tuant un serpent ; c'est seulement grâce à l'oiseau reconnaissant que le héros pourra revenir sur terre ${ }^{32}$. Dans notre čörčök, l'oiseau et le héros échangent plutôt un signe de vie (motif E761). L'oiseau est nommé Qaan-Kerede : ce n'est autre que l'oiseau Garuda qui figure dans de nombreux récits du folklore d'Asie centrale et de Sibérie (iakoute Xarday, touvinien Xereti, mongol Xangarid, bouriate Xerdig) :

Au nombre des «tâches difficiles" que doit accomplir le héros du conte et de l'épopée, on compte aussi le voyage chez l'oiseau Garuda, qui vit dans un pays extrêmement lointain. L'oiseau Garuda est en conflit avec un serpent qui mange ses petits (lesquels sont parfois représentés sous forme anthropomorphe comme de jolies filles). Un serpent gigantesque (Avraga Mogoy, par exemple) sort de l'océan pendant l'absence de l'oiseau Garuda et attaque son nid. Le héros tue le serpent et l'oiseau reconnaissant devient l'auxiliaire du héros (voir les sujets analogues relatifs aux oiseaux Qaraqus ou Simourgh chez les peuples turcs). ${ }^{33}$

19 (Ajoutons que Qaraqus est chez les Altaïens [Téléoutes] le patron des chamanes, qu'il aide lors de leurs voyages dans le monde des esprits ${ }^{34}$.)

Avec le personnage de Qaan-Kerede, nous passons du conte de fées au mythe. Alors que les motifs liés à l'oiseau Garuda sont, pourrait-on dire, ambigus - ils peuvent se trouver 
dans le conte populaire aussi bien que dans le conte mythologique -, le personnage du castor est lui tout à fait mythique. Le čörčök commence par un beau passage parallélistique :

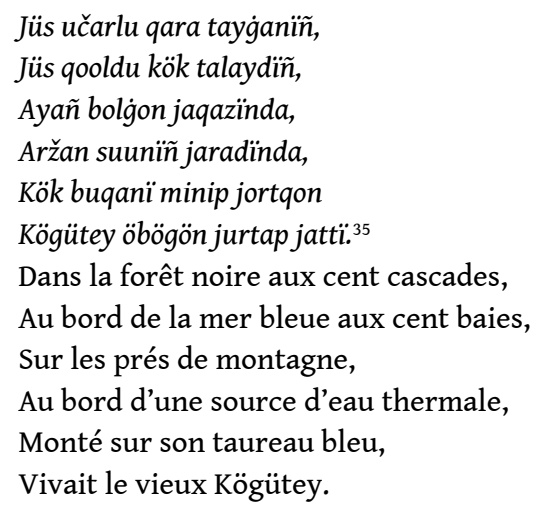

Dans ce passage (qui est d'ailleurs stéréotypé), une opposition entre terre et eau (forêt/ mer, pré/source, noir/bleu) s'ébauche, opposition qui prendra sens plus tard lorsque le castor, changé en homme, se révélera être le fils de " la Forêt noire aux cent cascades » et de "la Mer bleue aux cent baies». On pourrait aussi dire qu'il est le fils de la terre (jer) et de l'eau (suu). Le castor n'est donc pas seulement le héros sous forme animale du conte populaire, il est aussi une divinité chtonienne issue de la terre et de l'eau, deux éléments qui jouent un grand rôle dans la mythologie turque. C'est surtout quand ils sont liés que les mots yer (žer, jer, etc.) et suw (suu, etc.) prennent un sens religieux, yersuw dénotant le terrain sacré dans les textes en turc ancien. Dans la mythologie des Altaïens, jer-suu est la totalité des esprits supérieurs, des divinités bienfaisantes qui protègent les hommes ${ }^{36}$.

Alors que la trame narrative de Kögütey est caractérisée par la présence de deux séries de motifs, l'ensemble des motifs rencontrés dans Altay Buučay est beaucoup plus homogène. Cette épopée est connue de toutes les tribus turques altaïennes; Surazakov en cite treize variantes ${ }^{37}$. Je donne le résumé de la version du barde A. G. Kalkin, qui fut notée en 1958 par Surazakov. Elle compte 1421 vers; le barde appelle le héros « Altay Bučìy » et non « Altay Buučay »".

Altay-Buučay vit avec sa femme Jara-Čečen et sa propre sœur Očo-Čečen sur les prés de montagne et au bord de la mer. Un jour, il part à la chasse, chargeant sa sœur et sa femme de garder les troupeaux. Comme il ne revient pas, sa femme, le jugeant mort, demande à des canards où elle pourrait trouver un nouveau khan. Les oiseaux nomment les frères Arnay et Čarnay, et Jara-Čečen leur écrit une lettre sur les ailes des canards. Cette lettre parvient aux frères, qui s'empressent d'aller chercher les deux femmes. Ils sont reçus chaleureusement et chargés de tuer AltayBuučay, au cas où il reviendrait. Bien sûr, Altay-Buučay revient, au cours d'une tempête de neige. Sa femme lui donne du vin empoisonné, mais le poison n'a aucun effet sur Altay-Buučay. Les deux khans, qui s'étaient cachés dans une fosse, sont alors priés par les femmes de lutter contre Altay-Buučay. Mais au bout de sept jours ils n'ont toujours pas réussi à le vaincre. Altay-Buučay demande à sa femme et à sa sœur de jeter des pois sous les pieds des khans et de la farine sous ses pieds à lui. Les femmes font le contraire. Les khans peuvent alors enchaîner Altay-Buučay ; ils lui coupent les deux pouces, lui arrachent les yeux et, enfin, le tuent. Ils jettent dans une fosse son corps et celui de son fils, encore bébé, et s'en vont avec les femmes et les possessions d'Altay-Buučay.

Alors que la femme et la sœur d'Altay-Buučay, les vilaines, le trahissaient, ses trois chevaux - l'un pour la guerre, le deuxième pour la chasse et le troisième pour la garde des troupeaux -, ses deux chiens et ses deux faucons lui sont restés fidèles. 
Les faucons, changés en moineaux, trouvent les yeux et les pouces d'Altay-Buučay et les portent à son corps. Mais, malgré leurs efforts, les animaux ne parviennent pas à ranimer Altay-Buučay. Son fils cependant est encore en vie. Il part à la recherche de sa mère et de sa tante. Quand il parvient à la demeure d'Arnay et de Čarnay, il tue les meurtriers de son père, décapite sa tante et lie sa mère au poteau à chevaux. Rentré chez lui, il lit dans le livre de Sudur que seule la fille de Teñri-khan pourrait ranimer son père. Son cheval Temiči se change en aigle et vole au ciel avec le jeune héros. Le cheval attire l'attention de la fille de Teñri-khan, qui monte sur lui et descend sur terre. Là, elle redonne vie à Altay-Buučay et se marie avec son fils.

Un jour, Altay-Buučay part encore une fois à la chasse. Il rencontre un renard qui lui révèle où se trouve sa femme. Altay-Buučay va la chercher, l'accuse de trahison et lui crache trois fois au visage. Puis il s'en retourne, mais, avant qu'il ne soit rentré chez lui, une tempête se lève. Trois mangus, les patrons des khans Arnay et Čarnay, viennent de dessous la terre avec une armée de guerriers venger la mort de leurs protégés. Altay-Buučay tue un géant, dont la mort entraine celle de toute l'armée, puis d'une seule flèche il abat les trois mangus. Il peut enfin rentrer chez lui et jouir de la paix.

Dans ce čörčök on peut isoler quatre séries de motifs. La première se rapporte à la femme et à la sœur traîtresses : l'interdit énoncé par le héros (ici l'ordre de ne pas cesser de garder les animaux) ; la violation de l'interdit et l'invitation d'un homme (ou d'un monstre) malfaisant ; la trahison et le meurtre du héros. Des motifs secondaires apparaissent dans cette série, tels l'emploi des canards comme messagers ou l'interversion entre les pois et la farine jetés sous les pieds des combattants. La deuxième série de motifs concerne la réanimation du héros : d'abord par les animaux restés fidèles au héros, ensuite par le fils du héros qui redonne vie à son père, seul ou avec l'aide d'un personnage reconnaissant ou d'un être surnaturel. Après ces événements, le fils devient lui-même, le plus souvent, le héros de l'épopée; sa tâche principale, outre la réanimation de son père, est de tirer vengeance des meurtriers. Dans cette deuxième unité narrative s'intercale une quête matrimoniale : pour ranimer son père, le fils doit aller chercher l'aide de la fille de Teñri. Bien que la fonction première de cette divinité soit d'être un auxiliaire, le fils se marie avec elle, ce qui donne à son aventure l'aspect d'une quête d'épouse (peu développée, d'ailleurs). Dans Altay-Buučay, le meurtre est vengé deux fois, une fois par le fils et la deuxième fois par le père. Au lieu de narrer d'autres exploits du fils, la dernière partie de l'épopée voit à nouveau le père remis au premier plan. Il complète sa vengeance par l'humiliation de sa femme et l'anéantissement des mangus et de leur armée.

L'intrigue centrale de ce čörčök offre beaucoup de ressemblances avec nombre de motifs du folklore mondial. Mais celui de la mauvaise femme (ou sœur) est souvent développé d'une manière un peu différente (voir, par exemple, les types n $n^{\circ} 315$ et 590 dans l'index d'Aarne-Thompson). Les équivalents les plus proches se rencontrent dans le folklore mongol, en particulier dans les récits du type ${ }^{0} 94$ de l'index de László Lőrincz ${ }^{39}$. Ce n'est pas un hasard si ces récits mongols ressemblent à notre čörčök. C'est précisément chez les peuples mongols qu'existe une épopée si semblable à Altay-Buučay que l'on considère ces deux épopées comme issues d'une même source. Je parle de l'üliger bouriate Jerensej qui fut noté du barde Manšut Imegenov (Emegenej Manšuud) en 1905 par le mongolisant C. Žamcarano ${ }^{40}$. Cette épopée est beaucoup plus longue que le čörčök altaïen (9521 vers d'après l'édition de Xomonov), et il y a des différences de détail. Je ne signale que les plus importantes : le père (Jerensej) n'est trahi que par sa femme ; en plus d'un fils, il a aussi une fille, qui se marie dans la dernière partie de l'épopée ; le fils 
(Xanxan Sogto) réanime son père sans l'aide de la fille de Teñri ; le père passe au second plan et c'est désormais le fils qui est le héros de l'épopée ; il accomplit nombre d'actions héroïques, parmi lesquelles une quête matrimoniale (Nalxan Taiža); au cours de cette quête, il lui faut mener à bout plusieurs tâches, dont la dernière est l'obtention d'une plume de l'oiseau Garuda (Xanxan Xirdeg), aventure qui se déroule de la même façon que dans Kögütey.

D'après l'analyse de Roberte Hamayon, les üliger bouriates peuvent être classés en deux types principaux, les « épopées-à-sœur » et les « épopées-à-père ». Il y a aussi des soustypes, et Jerensej appartient à l'un des deux sous-types où se mêlent traits d'épopée-àsœur et traits d'épopée-à-père ${ }^{41}$. Sous cet éclairage, le monde narratif de l'üliger bouriate est un monde bien structuré qui se laisse interpréter, dans un cadre structuraliste, comme modèle poétique. Il n'y a pas lieu de donner ici un résumé de cette étude approfondie de l'üliger bouriate et des phénomènes associés au chamanisme. Dans le contexte de ce chapitre, il suffira de souligner la proximité entre épopées bouriate et altaïenne; on peut conjecturer que tout ce qu'on peut dire de l'épopée bouriate s'applique aussi plus ou moins à l'épopée altaïenne. En particulier, tout comme les üliger, les čörčök se laissent eux aussi interpréter dans le cadre de la Weltanschauung chamanique. N'étant pas un spécialiste du chamanisme, je me contenterai de revenir à la brève définition de Jean-Paul Roux donnée plus haut et d'énumérer les quelques traits des deux épopées, Kögütey et Altay-Buučay, en accord avec cette interprétation : "L'univers [est] conçu comme divisé en deux ou trois zones superposées, le ciel et la terre ou le ciel, la terre et le sous-terre, que relie un axe cosmique permettant le passage éventuel de l'une à l'autre; et l'existence d'une multitude de personnages invisibles mais zoomorphes, omniprésents dans les deux ou trois étages cosmiques et dans les régions qui les séparent, auxquels on peut donner par commodité le nom d'esprits. » Tandis que les trois mangus qui attaquent AltayBuučay, esprits tutélaires de ses meurtriers, viennent du monde souterrain, le fils d'Altay-Buučay se dirige vers le ciel, royaume de Teñri ${ }^{42}$. Le cheval favori d'AltayBuučay est d'ailleurs un cadeau d'Ülgen - selon la mythologie des Altaïens, le chef des esprits bienfaisants qui habitent le ciel, « celui qui meut le soleil et la lune $»^{43}$. Un autre élément chamanique, relié à la pratique extatique du chamane, est la guérison du malade dans sa forme la plus radicale, à savoir la réanimation du mort. Relève également du chamanisme la transformation du héros, de son cheval (et d'autres animaux), ainsi que de ses adversaires, rappelant la «transformation extatique » du chamane pendant son parcours des trois mondes ${ }^{44}$. La relation entre narration et vision du monde - sous ses aspects tant idéologique que sociologique - est plus profonde que ne le laisseraient supposer ces quelques éléments; mais ici nous nous contenterons de cette brève ébauche de l'épopée altaïenne.

L'étude de l'épopée orale turque archaïque nécessite d'ajouter quelques remarques sur l'épopée iakoute. Le sanskritiste Otto Böhtlingk a inclus un court oloñxo dans sa grammaire de la langue iakoute, parue à Saint-Pétersbourg en 1851. Ce petit texte en prose - Böhtlingk l'appelait un conte de fées (Märchen) - traite d'Er Sogotox, "l'homme solitaire ", l'un des héros primordiaux des Iakoutes. Il raconte sa lutte avec le gigantesque Buura Doxsun et son mariage avec Xotuuna, la plus jeune fille de Xara$\mathrm{khan}^{45}$. Nikolaj V. Emel'janov, qui a étudié les sujets de soixante-quinze oloñxo iakoutes, place ce récit dans le deuxième groupe de sa classification, celui des épopées sur les aïeux des Uraañxay Saxa (Iakoutes) ${ }^{46}$. En parcourant le livre de N. Emel'janov, on s'aperçoit aisément de la prédominance des données mythologiques dans ces épopées. 
Mais l'épopée iakoute est aussi riche en éléments chamaniques, comme l'a démontré A. Hatto dans une étude sur la trilogie Xaan Jarg̈istay, dans laquelle se trouvent trois scènes intimement liées aux idées et pratiques chamaniques ${ }^{47}$. Il est hors de doute qu'avec l'oloñxo iakoute nous sommes en présence d'une des formes les plus archaïques de l'épopée turque. Mais l'étude de ces récits et épopées outrepasse les limites imposées à ce chapitre.

Si l'on en revient à l'épopée des peuples turcs d'Asie centrale, on est d'abord frappé par la différence profonde entre, d'une part, les čörčök altaïens ou les oloñxo iakoutes et, d'autre part, les dastan ouzbeks, kirghizes, kazakhs, etc. Il faut naturellement écarter les romans d'aventure et d'amour et les épopées lyriques, d'origine plus récente et davantage liés à la littérature populaire du Moyen-Orient qu'à la mythologie d'Asie centrale et de Sibérie. Ce sont, bien entendu, les épopées héroïques qu'il faut comparer avec l'épopée altaïenne (et iakoute), telles Alpamiǐs, Qoblan, le cycle de Manas, Edige et autres. Ce qui distingue ces dastan héroïques des épopées archaïques de l'Altaï et de la Sibérie, c'est principalement leur historicité (ou souvent leur pseudo-historicité). Bien que de nombreux héros de ces épopées ne soient pas des personnages historiques, elles les présentent comme des figures qui ont leur place dans l'histoire. Elles plongent leurs racines dans l'histoire de la tribu, dans un passé qui se laisse souvent circonscrire: l'époque de la Horde d'Or (Edige), les temps de la Horde des Noghaïs (Qoblan) ou de la fédération tribale des Kiptchaks ou Qïpčaqs (Alpamǐ̌). Cette historicité n'est pas sans ambiguïté : s'il existe bien des racines historiques authentiques (ainsi, dans le cycle des héros des Noghaïs), on trouve aussi une historicité fictive, voulue par le barde et ses auditeurs. Tel est le cas du cycle de Manas, dont on a célébré le millénaire au Kirghizistan en 1995... Nous y reviendrons au chapitre suivant.

Par ailleurs, on relève beaucoup d'éléments fantastiques dans ces épopées, particulièrement perceptibles chez les adversaires du héros. Bien que ces figures soient issues de l'imagination populaire et souvent dotées de traits humoristiques, destinés à faire rire les auditeurs, on peut également reconnaître ici, me semble-t-il, les vestiges de l'imagination (et de la mythologie) sibérienne avec ses mangus et autre démons ${ }^{48}$. Arthur Hatto, dans son étude sur le chamanisme et la poésie épique dans l'Asie septentrionale, remarque à propos du héros kalmouk Almambet du cycle -kirghize de Manas :

In later texts, Almambet has the attributes of a weather-shaman. In conflict with his rival Chubak, in the words of Sagymbay, « Almambet squeezed himself, his face was all distorted... In his throat there was a cry... His nose grew sharp, his eyes sunken as a corpse's... From his mouth smoke poured out, from his eyes flames leapt crackling, his moustaches stood stiff as bodkins... Faster and faster he skipped, and the weather changed with a roar... A cloud was ripped open in the sky... Against the heavens all aflame Almambet loomed like a dragon. The storm then emptied itself in a fall of gleaming snow. Almambet's eyes grew large. A thunderous stormwind tore snarling from sunrise... a blizzard flew in squealing... » ${ }^{49}$

Arrivés à ce point, il nous faut revenir sur l'épopée karakalpake Qoblan. D'une manière très sommaire, on pourrait décrire la trame de ce dastan comme une série de luttes et de combats du héros contre des adversaires dont la plupart sont des êtres gigantesques et monstrueux. La seule exception dans cette série est la quête en mariage de la première partie de l'épopée. Ces monstres sont appelés däw, un mot (emprunté du persan dîv) qui, en karakalpak, désigne un géant et un ogre. Dans la version d'Esemuratžïraw ce sont : Alañgasar Älip-däw (aux dépens de qui Qoblan gagne son cheval), son frère Böke-däw, ensuite Köbikli-däw, le défenseur de la ville d'Azïwlï, et enfin la géante 
Däwkempir et son allié Šarïqlï-däw. Presque tous ces noms ont une signification : Alañgasar Älip-däw veut dire Ogre-Héros-stupide, Köbikli-däw Ogre-bavard, Däwkempir Ogresse-vieille, et Šarïqlï-däw Ogre-aux-chaussures-turkmènes. Le terme böke dans Böke-däw n'existe pas en karakalpak contemporain; le barde karakalpak Žumabay-žïraw, que j'ai interrogé sur le sens de ce mot, ne le connaît pas lui non plus. En kazakh, le mot n'existe pas. Mais, pour un mongolisant, il n'est pas sans signification. Dans une épopée oïrate du Xinjiang, Čerig-ün čaġan Qan (« Le khan-blancdes-guerriers »), récemment traduite en allemand par Klaus Koppe, on rencontre le personnage de Ġajar-un ġangġ-a böke ${ }^{50}$. Or, en mongol, böke (khalkha böx) veut dire le lutteur. Le terme existe aussi en kirghize (bökö, « lutteur ») ; la survivance de ce mot dans Qoblan démontre, à mon avis, la nature archaïque de nombre de ces figures-ogres, qui ne surgissent pas toutes de l'imagination individuelle du barde mais font apparemment partie de son héritage ${ }^{51}$.

Une autre caractéristique de l'épopée altaïenne est la fréquence de la transformation du héros et des autres personnages. De telles transformations sont beaucoup moins fréquentes dans l'épopée des peuples turcs islamisés, mais il s'en trouve quand même. Ainsi, le cheval de Köroğlu a dans une version azerbaïdjanaise la faculté de changer son aspect en celui d'une rosse quand le héros entre dans une ville, afin de ne pas provoquer le «mauvais œil » et exciter l'envie des gens ${ }^{52}$. Un autre trait de l'« épopée sibérienne ", la réanimation du héros, se rencontre aussi dans la poésie épique des Turcs d'Asie centrale ${ }^{53}$. Dans le cycle de Manas, le héros est ranimé à plusieurs reprises, comme j'en ai déjà fait la remarque dans le chapitre d'introduction. La version du cycle éditée par Radloff connaît deux occurrences de ce motif: la première, le héros est ranimé grâce à son faucon, à son cheval et à son chien, restés fidèles à leur maître même après sa mort ; la seconde, le héros empoisonné est ramené à la vie par sa femme Qanïkey :

Üydö džatqan Qanikey

Kerätindä bilät deyt.

Qan Temir dari alat deyt.

Manastï köstöp salat deyt.

2425 Kök-dosqoqtun astïnda

Batir tuugan Manasqa

Džetä kelip džattï deyt.

Qaynatma qara darïnï

Qaynatïp quydu džaraġa:

2430 Sürtmö qizil darïni

Sürtüp qoydu Manasqa.

Mekedägi Qan-qodžo

Ď̌etä keldi Manasqa.

Kün on eki bolgondo

2435 Tün on eki bolgondo,

Džaqši bolduñ, Er Manas $!^{54}$

Qanïkey, couchée dans la yourte,

Apprend d'une manière miraculeuse [la mort de Manas], dit-on.

Elle prend le médicament de Qan Temir [son père], dit-on,

Et cherche Manas, dit-on.

2425 En bas de Kök-dosqoq

Elle arriva, dit-on,

Auprès de Manas, héros dès sa naissance.

Elle fit bouillir et coula dans la plaie

Le médicament-bouillon noir : 
2430 Elle mit sur Manas

Le médicament-crème rouge.

Qan-qodžo de la Mecque

Vint aussi chez Manas.

Après douze jours,

2435 Après douze nuits,

Tu fus guéri, Er Manas!

31 Parmi tous les récits des traditions centrales, le plus proche du monde sibérien est probablement un autre poème kirghize. Le titre de la traduction française de ce texte (par Pertev Boratav) indique déjà cette proximité : Aventures merveilleuses sous terre et ailleurs de Er-Töshtük le géant des steppes (1965). Arthur Hatto en fait mention dans son étude du chamanisme et de la poésie épique en Asie septentrionale :

The familiar theme of the hero's journey to the Underworld, widespread in herotales of the Altai, was worked up to epic length in the Kirghiz poem of Er Töštük. Töštük is helped past many obstacles by specialists. He finally escapes to the Middle World on the back of the Cosmic Eagle, whose chicks he has saved from the Serpent at the foot of the World Tree. A highly shamanistic trait is that Töštük fuels the Eagle's flight with flesh from his own body. ${ }^{55}$

Étant donné ce que j'ai déjà dit du čörčök altaïen d'Altay-Buučay, il n'est pas nécessaire de s'étendre sur le motif de l'aigle reconnaissant. Les "spécialistes » que Hatto mentionne, sont des personnages, bien connus des contes de fées, qui possèdent chacun une faculté particulière portée à un degré miraculeux (comme de jeter des pierres plus loin que n'importe qui, de boire toute une mer d'eau, d'entendre les bruits les plus éloignés, etc.) ; ils deviennent les compagnons du héros et, le cas échéant, lui prêtent leur aide ${ }^{56}$. Er Töštük ne se laisse pas seulement rapprocher des épopées altaïenne et sibérienne, mais aussi de l'épopée anglo-saxonne de Béowulf. Malgré leurs différences profondes, ces deux traditions épiques assez éloignées ont un trait commun : leur sujet, qui n'est autre que celui du conte populaire de Jean de l'Ours (Aarne-Thompson, $\mathrm{n}^{\circ}$ 301). Si l'on compare ce conte de fées avec les épopées d'Er Töštük et de Béowulf, on peut constater que leurs différences les plus significatives ne se situent pas au niveau du sujet, mais plutôt à celui de la forme et du style ${ }^{57}$. Dans ces deux épopées, un récit archaïque, dont le motif central est la lutte du héros contre un ou plusieurs monstres du monde sous-terrain, est "épopifié ", c'est-à-dire développé en épopée. Ce développement comprend la description des personnages, l'élaboration scénique de l'action (monologues, dialogues, disputes, vantardise du héros et de ses adversaires, etc.), l'incorporation de scènes typiques (comme le départ du héros, le festin, la lutte héroïque, etc.) et certaines caractéristiques stylistiques. Béowulf n'est pas dépourvu de tout élément historique, mais malgré cette touche de "couleur locale " ni le héros central ni ses adversaires - deux monstres et un dragon - ne sont des personnages historiques! Il en va de même pour Er Töštük: bien qu'on l'ait rapproché du cycle de Manas, d'une manière assez artificielle, d'ailleurs, le récit ne laisse aucun doute, nous sommes au sein d'un monde fabuleux et merveilleux.

Si l'on s'adonne à l'«archéologie littéraire », plus on fouille profondément, plus la strate archaïque du chamanisme se laisse clairement reconnaître. Même des éléments religieux comme l'intervention des pir et la vénération des saints se révèlent être des survivances préislamiques. Le personnage du barde - son élection et sa vocation divine -, sa manière de chanter et son instrument de musique tracent aussi un lien avec le chamane préislamique. Et, comme on l'a vu, la présence de certains motifs et schémas narratifs laisse soupçonner une strate archaïque dans l'épopée héroïque des Kirghizes, 
des Kazakhs, des Ouzbeks, des Karakalpaks et des autres peuples turcs d'Asie centrale. Mais bien que les épopées des Turcs islamisés et non islamisés partagent cette même strate, il serait faux de négliger les différences entre ces traditions respectives. S'il est légitime de lire et d'interpréter les épopées des Turcs de l'Altaï et de Sibérie sur le plan du mythe, il me semble que la clé pour une compréhension adéquate de l'épopée héroïque des traditions centrales n'est pas le mythe mais plutôt l'histoire. Des figures comme Altay-Buučay ou Er Soġotox sont des ancêtres mythiques, conçus dans l'épopée comme des êtres primordiaux, existant hors du temps historique humain. En revanche, des héros comme Qoblan ou Alpamiiš sont replacés dans une époque historique concrète, même si cette localisation dans une période historique n'est pas celle de l'historien mais du poète. Avant d'aborder la question de la conception historique de l'épopée - question intimement liée à son interprétation -, je conclurai par une brève note sur l'épopée d'Alpamiš et sa version altaïenne.

Si les čörčök de l'Altaï ne partagent généralement pas les mêmes sujets que les dastan des Ouzbeks et d'autres peuples turcs d'Asie centrale, tel n'est pas le cas pour Alpamiš. Une variante de cette épopée fut notée en 1939 de la bouche du barde altaïen N. U. Ulagašev (1861-1946). D'autres versions altaïennes existent, recueillies en particulier dans la région septentrionale de l'Altaï, chez les Téléoutes et les Koumandine ${ }^{58}$. Le fil narratif de la version d'Ulagašev est comparable à celui qui caractérise les versions ouzbèkes, kazakhes et karakalpakes. Le héros, Alïp-Manaš, est emprisonné dans un pays ennemi ; il envoie un appel à l'aide par l'intermédiaire d'une oie sauvage; il est enfin libéré du cachot par son cheval; et quand le héros est de retour, il arrive juste à temps avant le remariage forcé de sa femme restée fidèle. Certes, il y a des divergences, surtout en ce qui concerne le mariage du héros. Quand Alïp-Manaš part gagner la main d'une vierge guerrière, Erke-Qaraqčii, il est déjà marié. Il est fait prisonnier en cours de route, durant son sommeil, et ne participe donc pas au concours des prétendants. Mais les différences sont beaucoup plus profondes entre les versions altaïennes et celles des Turcs islamisés. Dans le récit ayant pour héros AlïpManaš, les personnages et leurs actions sont dépeints comme dans l'épopée altaïenne : le héros est de stature gigantesque, son cheval vole au ciel et se transforme en étoile et plus tard en un mince cheveu, tandis que son ennemi, le traître Köböñ, s'enfuit changé en grue. On peut interpréter ces différences comme des traits d'un style commandé par les conventions du genre. Mais on peut aussi se demander si les versions n'ont pas conservé une forme plus archaïque de l'épopée. Viktor Žirmunskij a émis l'hypothèse que l'épopée d'Alpamǐs est née dans la partie méridionale de l'Altaï au cours des $\mathrm{VI}^{\mathrm{e}}$ $\mathrm{VIII}^{\mathrm{e}}$ siècles sous la forme d'un conte héroïque et a été diffusée à partir de là par les Oghouzes et plus tard par les Kazakhs ${ }^{59}$. Nikolaj Surazakov, en revanche, a exprimé l'opinion qu'il faut chercher les racines de l'épopée chez les Kiptchaks qui étaient au nord et à l'ouest de l'Altaï dans la période pré-mongole - il souligne que les Kiptchaks ne sont pas seulement un élément entrant dans l'ethnogenèse des Ouzbeks, des Kazakhs et des Karakalpaks, mais constituent aussi une tribu importante des Altaïens ${ }^{60}$. Cette question de l'origine est compliquée, et peut-être insoluble. La discussion sur les versions différentes d'Alpamiš montre que la recherche de strates archaïques dans l'épopée turque tend à se transformer en une recherche des origines. Il serait prématuré de s'engager sur ce point. Je me suis donc contenté dans ce chapitre, lors de la discussion des archaïsmes (et traits chamaniques) dans l'épopée orale d'Asie centrale, d'attirer l'attention sur leur dimension génétique. 


\section{NOTES}

1. Le texte kazakh en écriture arabe se trouve dans l'appendice de l'ouvrage de Castagné 1930, G14 sq., et sa transcription en écriture latine p. 78 sq. Je donne le texte dans le système de transcription adopté ici. La traduction française se trouve p. 76 sq.

2. La traduction de Castagné donne « te » au lieu de « le », erreur que j'ai corrigée.

3. Cf. chap. II, n. 30.

4. La même métrique caractérise aussi les chants des chamanes mongols ; cf. Even 1988-1989, pp. 318-336.

5. Le texte est pris de l'édition de Ergin 1958-1963, I, p. 155, mais translittéré d'après le système adopté pour ce livre.

6. Sur ces küy, cf. Elemanova et al. 1993, p. 95 sq.

7. Cette ligne provient d'une version versifiée de la légende, notée chez les Kazakhs du Xinjiang (par Mäžit Äbuwzar) ; cf. Baybulat et Balïqšï-ulï 1987, pp. 1-9.

8. Žarqïnbekov 1987, pp. 33-37 ; cf. aussi Castagné 1930, pp. 61-63 ; selon cette version, Qorqut est l'inventeur du premier qobïz.

9. Castagné 1930 , p. 80/p. 81 ; voir aussi les poèmes chamaniques kazakhs publiés par Radloff 1893, II, p. 63 sq.

10. Au sujet du chamane kazakh, voir aussi la collection de textes publiée par Däwrenbekov et Tursïnov 1993. On y trouve également des extraits (en traduction kazakhe) d'un travail de Čokan Valixanov sur les traces de chamanisme chez les Kazakhs (cf. Valixanov 1958, pp. 144-176) et d'un essai de Ä. Margulan sur les légendes de Qorqut (pp. 99 sqq.).

11. Sur le mot burxan, etc., en ancien ouïghour, cf. Räsänen 1969, p. 89 (s.v. burxan).

12. Le mot désignant le chamane est qam en turc ancien, kām en moyen turc, xam en touvinien, qam en altaïen ; cf. Räsänen 1969, p. 228 (s.v. Kām) ; cf. Gabain 1974, p. 354 (s.v. qam). En iakoute, le terme pour chamane est oyuun.

13. Roux 1984b, pp. 62 et 63.

14. Šternberg 1908, p. xi.

15. Sur le chamanisme en général, cf. Eliade 1968; sur le chamanisme des peuples turcs et mongols, cf. Harva 1938, pp.449-561; Roux 1984b, pp.59-98; sur le chamanisme sibérien, cf. Hamayon 1990; Novik 1984; sur le chamanisme des peuples turcs, cf. Alekseev 1984; Chadwick 1932-1940, III, pp. 192-218; Radloff 1893, II, pp. 1-67 ; voir aussi les recueils de textes de Ksenofontov 1928 et Marazzi 1984. Une riche collection de chants de chamanes mongols a été éditée, traduite et commentée par M.-D. Even (1988-1989). Une analyse des pratiques chamaniques des Ouïghours se trouve dans un recueil d'articles sur le chamanisme, numéro 26 des Études mongoles et sibériennes (cf. Du Shaoyuan 1995).

16. Sur ces visions, cf. Žirmunskij 1979 ; sur les aşık turcs de Turquie, cf. Başgöz 1967.

17. Kydyrbaeva 1984, pp. 114-115; voir aussi les autres textes réunis pp. 111-117.

18. "Wherever singing is an act of ecstasy and depersonalization, it moves away from ordinary human expression » (Sachs 1962, p. 83).

19. Cf. Rouget 1990, p. 239 sqq.

20. En russe, cette manière de chanter est appelée gorlovoe penie ; cf. Šul'gin 1973.

21. Hamayon 1990, pp. 590-591.

22. Cf. Zerańska-Kominek 1992, p. 312. J'ai décrit la manière de chanter du baxši ouzbek dans Reichl 1985a, p. 616 sqq. Il faut ajouter que cette manière de parvenir au " point d'ébullition »se trouve aussi dans le maqām (sorte de suite) de la musique classique ouzbèke, où l'on parle de « point culminant » (audž, mot arabe); cf. Veksler 1965, p. 217 sq. 
23. Il est difficile d'être sûr de l'authenticité de nombre d'enregistrements et de se faire une impression correcte de la qualité des voix; je renvoie par exemple aux numéros 7 (« chant de chamane des Toungouses-Nanaï»), 11 («chant de chamane des Toungouses-Oultch») et 17 ("chant de chamane des Nganasan ») du disque Musique de la toundra et de la taïga (URSS), dont seul le dernier numéro permet d'entendre chez l'un des chanteurs cette qualité de voix dite « resserrée » ou étranglée (Maison des cultures du monde, 1987 et 1990, coll. Inédit).

24. Castagné 1930, p. 67.

25. Ces peuples sont les Altaï-kiži, les Téléoutes et les Tölös, les Télenghites, les Koumandines, les Yiš-kiži et les Quu-kiži ; cf. Pritsak 1959 ; Baskakov 1966.

26. Cf. Radloff $1866 b$.

27. Cf. la liste dans Surazakov 1985, pp. 242-255.

28. Le texte altaïen est édité, avec coupures, par Surazakov 1958, pp.86-129; une traduction russe plus complète est celle de Tokmašov et Zazubrin 1935 ; cf. Surazakov 1985, p. 244, nº 31.

29. Les traits les plus saillants qui marquent le texte comme épopée sont la structure métrique (vers allitérés plutôt que prose), le parallélisme syntaxique et les "scènes typiques ", en particulier la description du héros, de son armure et de son cheval.

30. Cf. Aarne et Thompson 1961 ; Swahn 1955.

31. Cf. Thompson 1955-1958; pour un conte populaire karakalpak dans lequel on trouve les mêmes motifs, cf. Reichl 1985c, pp. 26-27/pp. 67-69.

32. Voir aussi les motifs 12 sq. dans le type $n^{\circ} 72$ (Der Phönix) de Eberhard et Boratav 1953, pp. 78-82.

33. Tokarevet al. 1980-1982, I, p. 267 (s.v. Gáruda, pp. 266-267).

34. Cet oiseau est aussi l'auxiliaire du chamane chez les Kazakhs et quelques groupes d'Ouzbeks ; cf. Tokarev et al. 1980-1982, I, p. 622 (s.v. Karakús).

35. Surazakov 1958, p. 86. À propos de ce passage, voir une étude du style parallélistique dans les épopées turque et serbo-croate dans Reichl 1994.

36. Cf. Roux 1984b, pp. 132-141, surtout pp. 133-134 ; Tokarev et al. 1980-1982, I, p. 599 (s.v. Yer$\mathrm{Su})$.

37. Cf. Surazakov 1985, pp. 120-123.

38. Cf. Surazakov 1961, pp. 8-9; le texte se trouve pp. 79-123. Buučay est, selon Surazakov, une dérivation du terme mongol et bouriate buuč, « tireur ».

39. Cf. Ló'rincz 1979, p. 63.

40. Cf. l'édition de Xomonov 1968.

41. Cf. Hamayon 1990, p. 198 sq.

42. Cf. Tokarev et al. 1980-1982, II, pp. 500-501 (s.v. Téngri). Pour une comparaison du chamane et du barde comme médiateurs, cf. Hamayon 1981, p. 207 sq. ; 1990, p. 590 sq.

43. Cf. Tokarev et al. 1980-1982, II, pp. 546-547 (s.v. Ul'gén'). Sur la mythologie des peuples turcs, voir aussi l'article Tjurkojazyčnyx narodov mifologija, ibid. II, pp. 536-541.

44. Sur la transformation du héros épique, cf. Chichlo 1981.

45. Cf. Böhtlingk 1851 [1964], pp. 79-95 (texte iakoute et traduction allemande). Le lecteur français peut se familiariser avec l'épopée iakoute par une traduction récente de trois récits iakoutes ; cf. Karro et Sabaraikina 1994.

46. Emel'janov 1980, n 17 (pp. 76-81); pour les autres versions, cf. ibid. $\mathrm{n}^{\circ}$ 18-25 (pp. 81-119).

47. Cf. Hatto 1985.

48. Cf. Reichl 1992, p. 289 sq.

49. Hatto 1980, p. 137.

50. Cf. Koppe 1993, pp. 69-109.

51. Le terme mongol böke correspond aussi au tchaghataï bökä, « lutteur », altaïen pökö, touvinien mögö, iakoute bögö, «fort»; il ne semble pas apparenté au mongol böge (khalkha böö), « chamane », dont il existe aussi des équivalents dans les langues turques. Cf. Räsänen 1969, p. 83, 
s.v. bökä, bögü. Sur la relation entre mongol classique bökö « (böke) lutteur » et bögö « (böge) chamane », cf. aussi Hamayon 1990, p.142. Il est bien probable que d'autres personnages fantastiques des épopées turques, comme les peri, les sorcières yalmāàuz et māstān, les lutteurs chauves ( $k a l)$, etc., proviennent d'une couche archaïque de la tradition épique.

52. "That wonderful horse possessed also this peculiarity, that whenever he came to any strange city, he dropped his ears like an ass, made his bristles stand up on end, and with his mane dishevelled, his tail thrust between his legs, walked along like a jade, in order that none of the passers-by might covet him, or throw a spell on him » (Chodzko 1842, p. 97).

53. À ce sujet, cf. Heissig 1981 ; Hamayon 1981, p. 179 sq.

54. Hatto 1990, p. 298/p. 299.

55. Hatto 1980, p. 137.

56. Sur ces personnages, cf. Thompson 1955-1958, motif F601 sq. (Extraordinary companions).

57. Cf. Reichl 1987.

58. Cf. Surazakov 1985, p. 67 ( $\left.\mathrm{n}^{\circ} 41,178,184,209\right)$. La version d'Ulagašev est publiée dans Surazakov et al. 1959, pp. 28-67 ; voir le résumé dans Žirmunskij 1960, pp. 140-144.

59. Cf. Žirmunskij 1960.

60. Cf. Surazakov 1959.

\section{RÉSUMÉS}

Dans le troisième chapitre, les strates pré-islamiques rencontrées dans les épopées d'Asie centrale sont examinées de plus près. On montre l'existence d'une étroite connexion entre le barde et le chamane. Celle-ci est mise en évidence par l'usage de termes comme baxši pour désigner l'un comme l'autre, le symbolisme de l'instrument du barde, comparable à celui du chamane, les visions et la maladie initiatique dont ils font tous deux l'expérience. Une étude plus poussée de deux épopées altaïennes (Kögütey et Altay Buučay) montre que cette tradition a bien préservé le monde des Turcs pré-islamiques, mais des strates archaïques semblables peuvent aussi être détectées dans les épopées des traditions centrales, parmi lesquelles on peut mentionner les transformations du héros et de son cheval, les aventures hérö̈ques dans le monde souterrain, diverses figures mythologiques et la réanimation du héros.

In the third chapter the pre-Islamic strata as found in the Central Asian epics are further examined (Le héros et le chamane: les strates archaïques de l'épopée turque). It is shown that there is an intimate connection between epic singer and shaman. This emerges from the use of terms like baxši for both the bard and the shaman, from the symbolism of the singer's instrument, comparable to that of the shaman, and from initiation visions and sicknesses found both among bards and shamans. A closer view at two Altaian epics (Kögütey and Altay Buučay) shows that in this tradition the world of the pre-Islamic Turks is well preserved, but similar archaic strata can also be detected in the epics of the central traditions, among them the transformations of the hero and his horse, heroic adventures in the underworld, various mythological figures and the reanimation of the hero. 
INDEX

Mots-clés : Asie centrale, barde, chamane, épopée, héros

Thèmes : islam, littérature, mythologie

nomsmotscles Karakalpak, Kazakh, Kirghiz, Ouighour, Ouzbek, Turc, Yakoute

\section{AUTEUR}

KARL REICHL

Karl Reichl est professeur de philologie anglaise à l'Université de Bonne (Allemagne). Il a publié de nombreux travaux sur la littérature orale des peuples turcs : Turkic Oral Epic Poetry: Traditions, Forms, Poetic Structure (1992), The Oral Epic: Performance and Music (2000), Singing the Past. Turkic \& Medieval Heroic Poetry (2000), Edige: A Karakalpak Oral Epic (2007). 